\title{
Controlling arbitrary humidity without convection
}

\author{
Priyanka S. Wasnik, Hartmann E. N'guessan and Rafael Tadmor* \\ Dan F. Smith Dept. of Chemical Engineering, Lamar University, Beaumont, TX 77710, United States
}

\begin{abstract}
In this paper we show a way that allows for the first time to induce arbitrary humidity of desired value for systems without convective flow. To enable this novelty we utilize a semi-closed environment in which evaporation is not completely suppressed. In this case, the evaporation rate is determined both by the outer (open) humidity and by the inner (semi-closed) geometry including the size/shape of the evaporating medium and the size/shape of the semi-closure. We show how such systems can be used to induce desired humidity conditions. We consider water droplet placed on a solid surface and study its evaporation when it is surrounded by other drops, hereon "satellite" drops and covered by a semi-closed hemisphere. The main drop's evaporation rate is proportional to its height, in agreement with theory. Surprisingly, however, the influence of the satellite drops on the main drop's evaporation suppression is not proportional to the sum of heights of the satellite drops. Instead, it shows proportionality close to the satellite drops' total surface area. The resultant humidity conditions in the semi-closed system can be effectively and accurately induced using different satellite drops combinations.
\end{abstract}

KEY WORDS: Evaporation suppression; Controlled humidity; surface phenomena; Satellite drop; Evaporation rate; Relative Humidity; Evaporation flux; Maxwell Equation; Rowan Equation

(C) 2015. This manuscript version is made available under the Elsevier user license http://www.elsevier.com/open-access/userlicense/1.0/ 


\section{INTRODUCTION}

Methods for arbitrary control of evaporation rate have many applications including drying ${ }^{1,2}$; cooling $^{3,4}$; membrane separation ${ }^{5}$; thin-film deposition ${ }^{6,7,8}$; nano-composite films ${ }^{9,10}$; biomedical applications $^{11,12,13}$; molecular biology ${ }^{14}$; food engineering ${ }^{15,16}$; geological science ${ }^{17}$; and materials ${ }^{18}$, just to name a few. A number of methods have been introduced for this end, and as a rule they utilize either solutions of different salts ${ }^{19}$, or, more commonly, saturated salt solutions $^{20,21,22}$ (the saturation maintains constant salt concentration in the solution and therefore produces constant humidity). There are various disadvantages to these methods: and the main one is the introduction of another chemical (the salt solution) which can contaminate the system $^{23,24}$ or corrode it or both. Other disadvantages include the need for different salt for each humidity, rendering a salt for a specific humidity potentially unavailable. Yet another problem with those methods is related to the actual practice of these systems: usually they are constructed in the lab, and the seal is reasonable but not perfect. To make sure that the desired humidity is maintained, typically air is bubbled through the salt solution, and carries with it the desired humidity to the actual system whose humidity we want to control and this flow overshadows any leakages that the system may have. The disadvantage is the need to create flow, and the added complexity of having a pump and an external big system to control humidity in a small system. As we shall see, in this paper we use basic principles of drop evaporations to overcome all these problems.

Drop evaporation has been studied extensively over the years ${ }^{25,26,27,28,29}$ often in relation to different evaporation modes, most notably the so called CCR (constant contact radius) and CCA (constant contact angle) modes ${ }^{30,31,32}$. The existence of a CCR mode is related to pinning, which 
emanates from intermolecular re-orientation of the solid that is enhanced at the triple line as has been described experimentally and via simulation ${ }^{31,32,33}$. While exceptions to this rule exist ${ }^{34}$, they will not affect the discussion of this paper.

Less attention, however, was given to evaporation suppression. Belman et al. ${ }^{29}$ suggested that the evaporation rate near the triple line of high contact angle drops is suppressed by the vicinity of the solid. Indeed, $\mathrm{Xu}$ et al. ${ }^{35}$ showed that the initial evaporation rate of a water droplet placed on a superhydrophobic surface is slowed down due to the large contact angle that results in a narrow wedge region of air along the contact boundary, where the liquid - vapor diffusion is restricted by the substrate. This results in an evaporation rate that is lower than predicted by Rowan model. In this paper we consider this understanding and choose a system in which the contact angle is close to $90^{\circ}$ in which the Rowan model is expected to be more accurate. Such understandings help us approach the subject of this paper, namely utilizing drop evaporation to control relative humidity and suppress evaporation.

Open system (an imaginary control volume without real boundaries to the air) and closed system (a confined volume completely isolated from the open air) will induce no control or complete suppression respectively, while a semi-closed system can induce desired arbitrary control. At the same time, to be effectively used, such systems require better characterization. This paper aims to address this need. We show that this approach can induce arbitrary humidity in a simple and practical way that is advantageous compared to other evaporation suppressing methods ${ }^{36,37}$. We study experimentally the evaporation of a water drop surrounded by other water drops referred to as "satellite" drops when the entire assembly is in a semi-closed environment. The satellite drops are placed equidistant from the central drop in different configurations. We show that the central drops' evaporation rate is the same within the experimental error when 
surrounded by satellite drops of the same total liquid-vapor surface area even if their other parameters such as number of satellites, their heights or volume, differ (namely different configurations of the satellite drops). However, the central drop's evaporation rate is different if surrounded by satellite drops configurations whose total sum of heights are the same but their total sum of surface areas is different (seemingly contradicting theoretical evaporation rates). Similarly, the central drop's evaporation rate is different if surrounded by satellite drops configurations whose total sum of volumes are the same but their total sum of surface areas is different. The evaporation of all these drops proceeds with a Constant Contact Radius (CCR ${ }^{25}$ mode during the time span presented in this paper except for drops placed in ambient air with no satellite drops in which case this mode is held only for the first 4 min.

In our group it has been reported that placing micro sized sessile water "satellite drops" around a central water drop produces effective evaporation suppression of the central drop ${ }^{38,39}$, nevertheless, a detailed study on this effect is performed here for the first time.

\section{Theory}

Maxwell ${ }^{40,41}$, showed theoretically that the evaporation rate, $\left(\rho \frac{d V}{d t}\right)$, namely the change of mass, $\rho V$, with time, $t$, of a spherical liquid drop of density $\rho$ and volume $V$ suspended in air, follows equation (1):

$$
\frac{d V}{d t}=-\frac{4 \pi R D M P_{o}(1-R H)}{\rho R_{g} T}
$$


where $R$ is the liquid drop radius; $D$ the liquid-vapor diffusion coefficient; $M$ the molecular weight of liquid; $P_{o}$ the saturated pressure of the liquid drop at its temperature $T ; R H$ the prevailing relative humidity and $R_{g}$ the universal gas constant. For the case of a drop on a surface Equation (1) was further modified by a few different theoretical studies (see nice summary by Erbil $^{30}$ ). Of these, we use here the expression by Rowan et al. ${ }^{42}$ as described in equation (2). The Rowan expression is accurate when the sessile liquid drop has a contact angle, $\theta$, in the vicinity of $90^{\circ}$. This is close to our system when a drop is placed on a flat surface in ambient air ${ }^{30,42,43,44}$. The evaporation rate at relative humidity $R H$ for this case is given by:

$$
\frac{d V}{d t}=-\frac{2 \pi h D M P_{o}(1-R H)}{\rho R_{g} T}
$$

where $h$ is the drop's height.

Our experiments were conducted under a hemispherical optical glass dome of $R_{D}=23 \mathrm{~mm}$ radius. Performing the integration over $R_{D}$ in case of Rowan equation, instead of Rowan et al.'s open system, results in equation (3) below:

$$
\frac{d V}{d t}=-\frac{2 \pi h D M P_{o}(1-R H)}{\left(1-\frac{R}{R_{D}}\right) \rho R_{g} T}
$$

where $R$ is the radius of the sphere the drop is part of, $R_{D}$ is the hemisphere (dome) radius, and $h$ is the drop's height. 


\section{EXPERIMENTAL SECTION}

\section{Materials}

Silicon wafer were obtained from Virginia Semiconductor, VA (diameter: $76.2 \mathrm{~mm} \pm 0.3 \mathrm{~mm}$, orientation: $<110> \pm 0.9^{\circ}$, dopant: Boron, resistivity: 0.0034-0.0046 $\Omega$-cm, center thickness: 381 $\mu \mathrm{m} \pm 25 \mu \mathrm{m})$ and cut into rectangular slides of $2 \mathrm{~cm} \times 2 \mathrm{~cm}$. Hydrogen peroxide (50 wt\% in water) was obtained from Acros Organics. Ethanol (99.5\%, 200 proof absolute), distilled water (0.1 $\mu \mathrm{m}$ filtered Molecular Biology Reagent), Ammonium Hydroxide (99.99\%), Hydrochloric acid (37\%), Toluene (99.5\%) and Octadecyltrimethoxysilane (90\% technical grade, CAS No. 3069-42-9) were obtained from Sigma Aldrich and used for self-assembly process ${ }^{45}$.

\section{Instruments}

The UV Ozone Cleaner used was Procleaner ${ }^{\mathrm{TM}}$ from Bioforce Nanoscience. The oven used to bake the surfaces was Cole Parmer Model 5053-10. Humidity measurements were done with a hygrometer pen (Extech Instruments) from Cole Parmer. The goniometer used was from Wet Scientific. It includes: a hemispherical optical glass dome (internal radius and height of $23 \mathrm{~mm}$ with a thickness of $2 \mathrm{~mm}$ ) fitting into a $1 \mathrm{~mm}$ deep $3 \mathrm{~mm}$ wide Teflon covered groove in the sample holder, and the related software for image processing from which geometric data (contact angle, height and diameter are measured) were obtained and verified manually.

\section{Procedure}

Step 1: Surface hydrophilization

The silicon wafers were made hydrophilic in one of the two methods below (both produced the same result).

Method 1 (UV/Ozone Cleaning): The Silicon wafer substrate was rinsed in a copious amount of ethanol followed by distilled water and then dried in the oven at $100{ }^{\circ} \mathrm{C}$ for 15 minutes. From 
the oven the substrate was transferred to the UV/Ozone Cleaner for 45 minutes to remove any organic contaminants on the substrate. Alternatively, RCA $^{\circledR}$ cleaning method can also be used.

Method $2\left(\mathrm{RCA}^{\circledR}\right.$ Cleaning $\left.{ }^{46,47}\right)$ : To remove contaminants and unwanted particles (dust, silica, metals), the wafer was dipped in $\mathrm{NH}_{4} \mathrm{OH}: \mathrm{H}_{2} \mathrm{O}_{2}: \mathrm{H}_{2} \mathrm{O}(1: 1: 5 \mathrm{v} / \mathrm{v} / \mathrm{v})$ at $70{ }^{\circ} \mathrm{C}$ for $15 \mathrm{~min}$. Then, the surface was washed with DI water before being immersed in $\mathrm{HCl}: \mathrm{H}_{2} \mathrm{O}_{2}: \mathrm{H}_{2} \mathrm{O}(1: 1: 6 \mathrm{v} / \mathrm{v} / \mathrm{v})$ at 70 ${ }^{\circ} \mathrm{C}$ for $5 \mathrm{~min}$. After this, the surface was dipped in toluene three times for $30 \mathrm{~s}$ to create an organic environment for the silanization process.

Both methods gave the same final results.

\section{Step 2: Silanization process}

Silanization took place by self-assembly process in a closed beaker. The silicon wafer substrate from step 1 was immediately immersed in a solution of 1 volume \% Octadecyltrimethoxysilane with 99 volume $\%$ toluene. The substrate remained in the solution, heated up to $70{ }^{\circ} \mathrm{C}$ for 3 hours ${ }^{48}$. The surface was then washed with water several times to remove any traces of toluene left on the surface before baking it in the oven at $100{ }^{\circ} \mathrm{C}$ for 15 minutes.

Step 3: Experimental Procedure

The experiments were conducted when the lab environment was at $25 \pm 1{ }^{\circ} \mathrm{C}$ and $32 \pm 4 \%$ Relative Humidity in a dust free laminar flow hood from Terra Universal ${ }^{\circledR}$ (ULPA filters). The silanized silicon surface was placed on top of the goniometer sample holder as shown in Figure 1. The water drops (central and satellite) were dispensed on the surface and immediately covered with the hemispherical optical glass dome. 


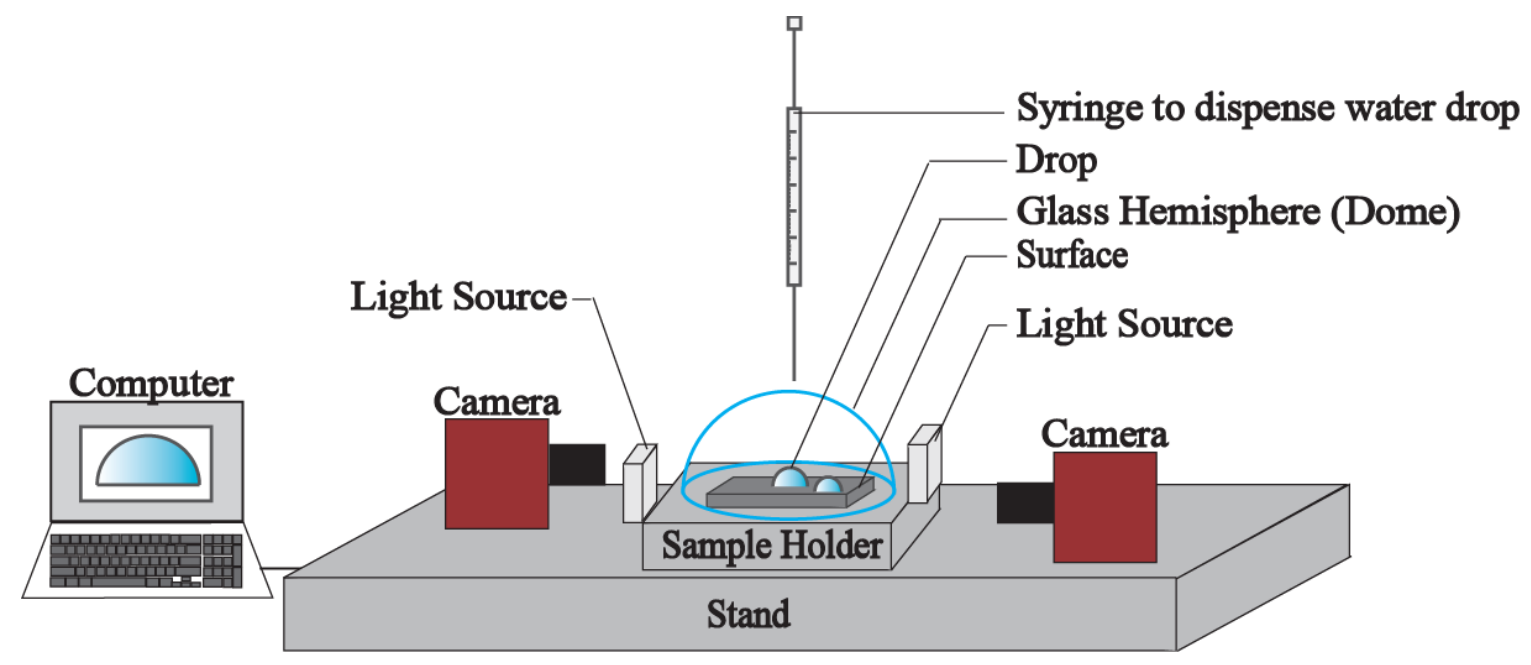

Figure 1. Schematic of the experimental assembly.

In our experiments we did not use the dome holder to press the dome to its groove because by doing so the evaporation is suppressed to impede timely study of evaporation. But if the dome rests in the groove only with its own weight, the seal is enough to induce a unique environment inside the dome, which is a clear function of the size and number of drops under the dome, and also loose enough for leakage through the groove to allow monitoring a timely evaporation. The area available for leakage affects equation 3 via the relative humidity term there $(R H$ in equation 3). The wider the gap size, the faster is the vapor transport between the inside of the dome and the lab, and hence the lower is $R H$. If the leakage area $\rightarrow \infty, R H$ equals that of the lab and if the leakage area $=0$ then the steady state relative humidity is $100 \%(R H=1$ in equation 3$)$. For the results to be consistent, there is a need to verify that the area available for leakage is similar for different experiments. In this study, this was done by verifying similar $R H$ values using equation 3 for different experiments with similar conditions, and at the same time, different $R H$ values using equation 3 when the conditions inside the dome are different. While maintaining the same 
leakage area is a requirement for our study, it is easily achieved by placing the dome with its own weight.

In all experiments we placed satellite drops of different volumes around a central drop of $1 \mu 1$. The number of satellite drops and their geometrical parameters (volume, surface area, height and location with respect to the central drop) is referred to as a "configuration". We found that the distance between the central and satellite drops does not influence the evaporation rate (for example, the evaporation rate of a central drop is the same when the satellite drops are $4 \mathrm{~mm}$ away from it or $8 \mathrm{~mm}$ away from it), The experiments reported here correspond to satellite drops placed at $4 \mathrm{~mm}$ away (center to center distance) from the $1 \mu \mathrm{l}$ central drop (which itself is about $0.77 \mathrm{~mm}$ in radius). The central drop was then monitored for changes in its volume over time. Examples of central drop pictures are shown in Figure 2.

Every point in the plots presented in this paper is an average of three experimental data points. All experiments with satellite drops were conducted under the dome.

\section{RESULTS AND DISCUSSION}

Using images such as those shown in Figure 2, we monitored the evaporation process in various configurations.

We study the evaporation of a $1 \mu \mathrm{l}$ central drop in the vicinity of different numbers $(1,2,4$, and 8) of satellite water drops such that the system (central + satellite drops) has the same initial sum of either: (1) volumes; or (2) surface areas; or (3) drop heights. 


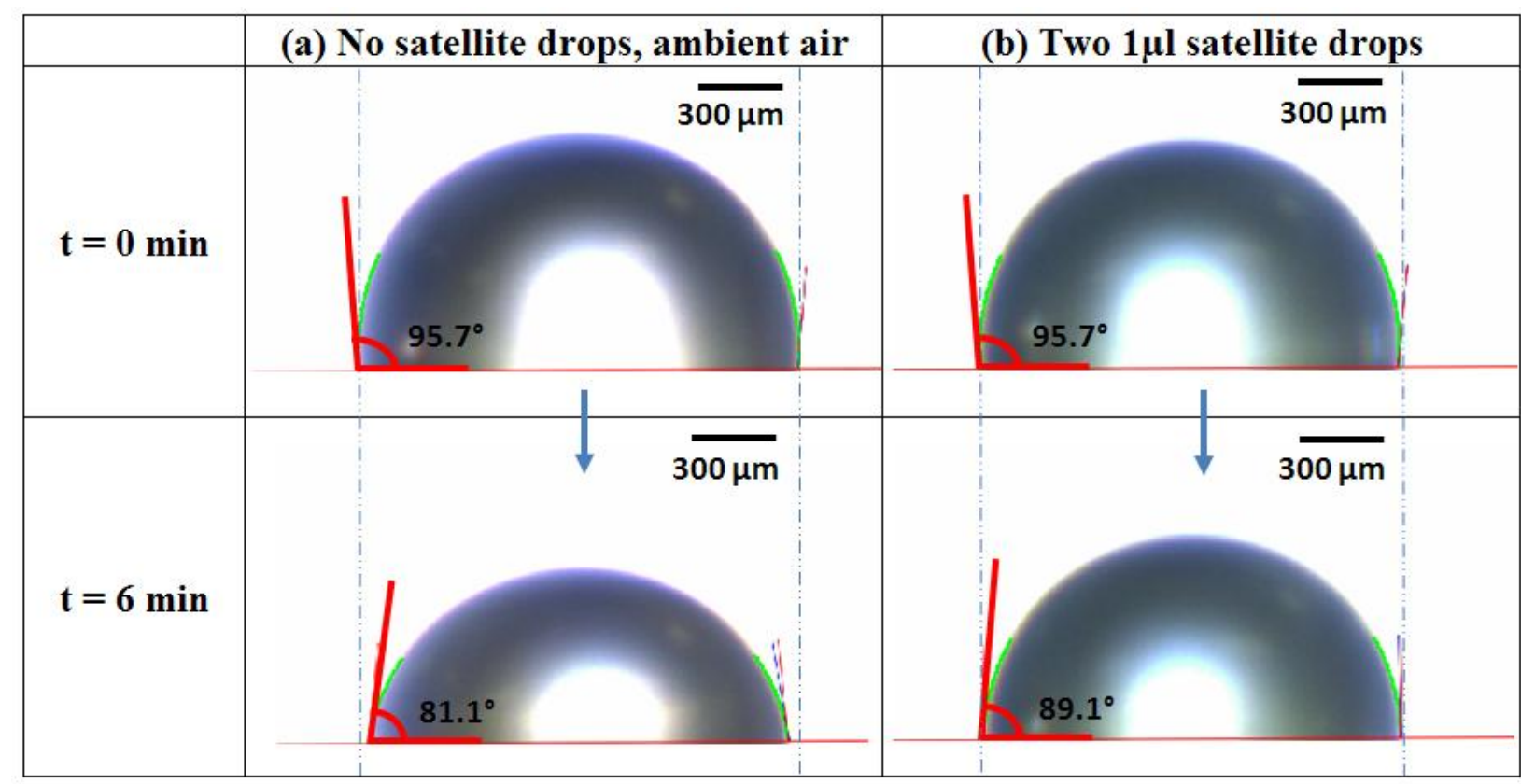

Figure 2. Frames from the evaporation process of $1 \mu \mathrm{L}$ central drops when it is either (a) open to ambient air, or (b) surrounded by two $1 \mu \mathrm{l}$ satellite drops and covered with $23 \mathrm{~mm}$ radius dome.

\section{Central drop volume as a function of time}

Figure 3 shows evaporation rates versus time for three cases. In each of the cases, the number of satellite drops change but are arranged so that the initial sum of a different geometrical parameter of them is the same. Specifically, the initial sums of either (a) volume, (b) area, or (c) length (sum of heights), when this sum is composed or 1,2, 4,or 8 satellite drops, are the same. In the case of figure $3 \mathrm{c}$ (sum of heights), the case of 8 satellite drops does not appear because the volume is too small to control reproducibly (each of the 8 small drop should have a volume that is $1 / 8^{3}=1 / 512$ times the volume of the single drop that is 8 times higher).

Four of the six curves in Figure 3a correspond to evaporation of a central drop when the satellite drops around it are different configurations of the same total volume of $2 \mu \mathrm{L}$. The two additional curves are control experiments with no satellite drops: one under the dome and one with no dome. Of the four that are not controls, note that the slowest evaporating central drop is 
the one surrounded by the biggest number of satellites (eight in this case), and hence the smallest size satellites $\left(0.25 \mu \mathrm{L}\right.$ or $7.7 \mathrm{~mm}^{2}$ each). Figure $3 \mathrm{~b}$ shows that when the constant parameter of the satellite drops is their total surface area the central drop evaporation rate is roughly the same regardless of their configuration. On the other hand, when the satellites constant parameter is their sum of heights (Figure 3c), the slowest evaporation is when the central drop is with a single satellite drop. A cross comparison between Figure $3 \mathrm{a}, 3 \mathrm{~b}$ and $3 \mathrm{c}$, therefore, shows that the central water drop's evaporation rate under the dome is a function of its surrounding satellite drops total surface area. An analysis of the central drop evaporation rate versus time shown in Figure $4 \mathrm{a}, 4 \mathrm{~b}$ and $4 \mathrm{c}$ gives a clearer insight into its evaporation rate mechanism.
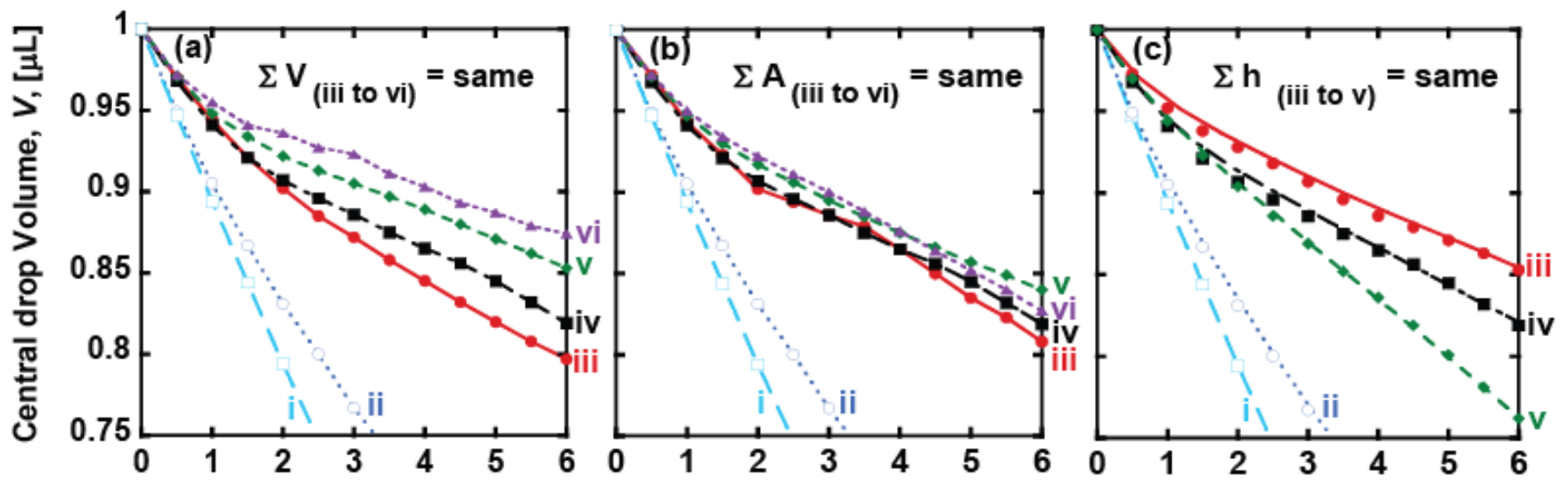

Time from drop placement, $t,[\mathrm{~min}]$

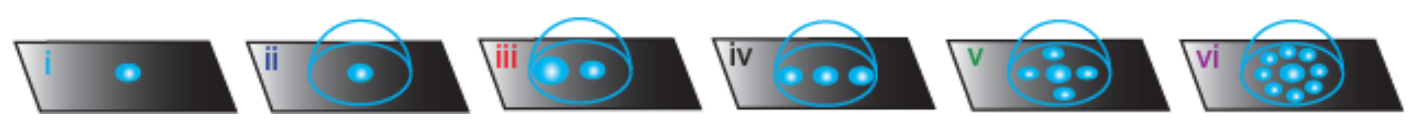

Figure 3. The volume, V, of a water drop on Silanized Silicon as a function of time when the drop is in: ( $\square$ ) Ambient air; (O) under the dome with no satellites; $(\mathbf{O}),(\square),(\diamond),(\boldsymbol{\Delta})$ under the dome with 1,2, 4 and 8 satellite drops respectively. The satellite drops have the same initial total sum of (a) Volume of $2 \mu \mathrm{L}$ (e.g. one satellite drop of $2 \mu \mathrm{L}$ or eight satellite drops of $1 / 4 \mu \mathrm{L}$ each). (b) Surface area of $7.7 \mathrm{~mm}^{2}$. (c) Heights of $1.6 \mathrm{~mm}$. The solid lines are guides to eye. The schematic of satellite drops under the dome are just for illustration purposes. In reality, the dome covers the whole surface as shown in Figure 1. The total surface area of satellite drops in 3 a iii, 
iv, $v$ and vi configurations are $6.0 \mathrm{~mm}^{2}, 7.8 \mathrm{~mm}^{2}, 9.8 \mathrm{~mm}^{2}$ and $12.4 \mathrm{~mm}^{2}$ respectively, while in $3 \mathrm{c}$ iii, iv and $\mathrm{v}$ configurations they are $15.9 \mathrm{~mm}^{2}, 7.8 \mathrm{~mm}^{2}$ and $4.0 \mathrm{~mm}^{2}$ respectively.

\section{Evaporation rate $(d V / d t)$ as a function of time}

Figure 4 shows that under the dome the central drop evaporation rate decreases with time for the first few minutes followed by a plateau. The time it takes for the system to reach a plateau decreases with the increase in the number of satellite drops when the parameter kept constant is the satellite drops' total volume (Figure 4a). An opposite observation is made by keeping the satellites sum of heights (Figure 4c) the same, where the time it takes to reach a plateau decreases with decreasing number of satellites. For configurations in which the satellite drops surface area are kept constant (Figure 4b), all the curves with satellite drops reach a plateau at a similar times. Once the central drop evaporation rate reaches a steady state, the vapor pressure under the dome increased to its maximal value and the evaporation rate to its minimal value. We also see that when the satellite drops total volume is kept the same ${ }^{49}$, the minimal plateau value for the highest number of satellite drops is lower as compared to cases of satellites of the same total surface area or the same sum of heights. We conclude that the higher the satellite drops total surface area, the lower is the evaporation rate change of the central drop and the faster its time to reach steady state. 

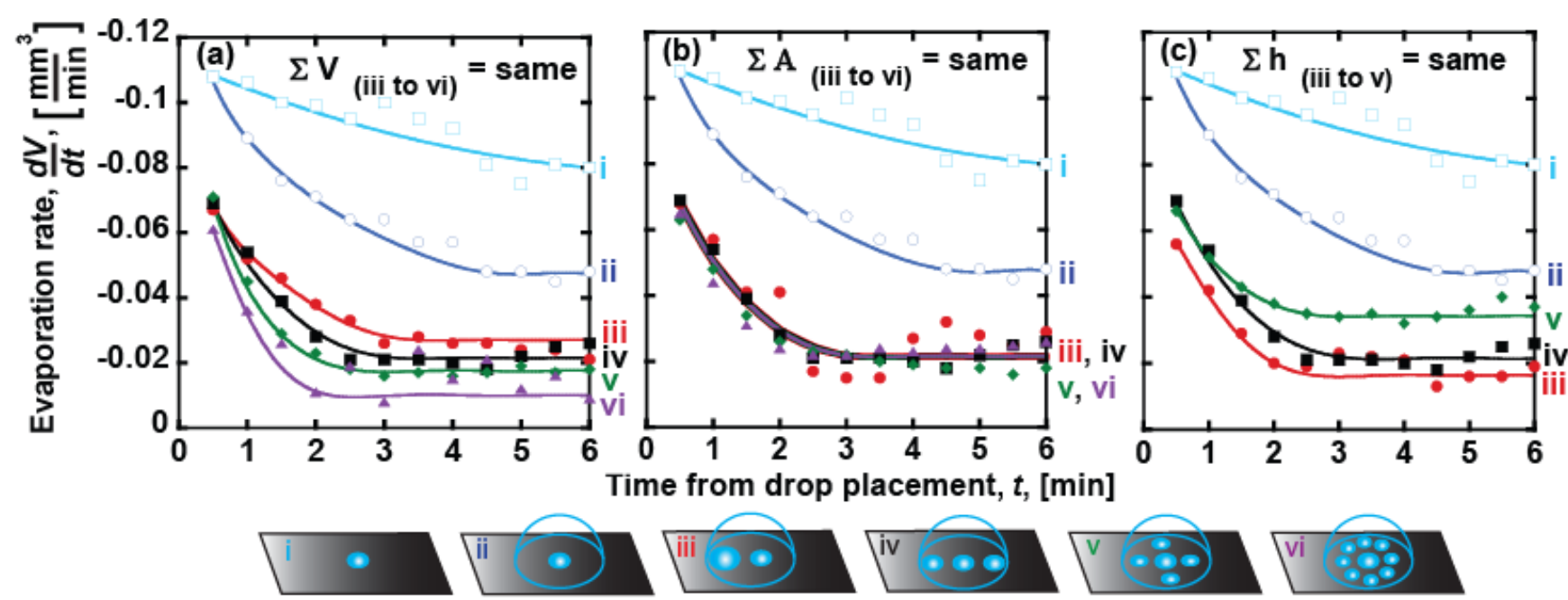

Figure 4. The evaporation rate, $\mathrm{dV} / \mathrm{dt}$, of a water drop on Silanized Silicon as a function of time when the drop is in: $(\square)$ Ambient air; (O) under the dome with no satellites; $(\bullet),(\mathbb{\square}),(\bullet),(\boldsymbol{\Delta})$ under the dome with 1, 2, 4 and 8 satellite drops respectively. The satellite drops have the same initial total sum of (a) Volume of $2 \mu \mathrm{L}$ (e.g. one satellite drop of $2 \mu \mathrm{L}$ or eight satellite drops of $1 / 4 \mu \mathrm{L}$ each). (b) Surface area of $7.7 \mathrm{~mm}^{2}$. (c) Heights of $1.6 \mathrm{~mm}$. The solid lines are guides to eye. The schematic of satellite drops under the dome are just for illustration purposes. In reality, the dome covers the whole surface as shown in Figure 1.

\section{Evaporation rate per area (flux, $d V / d t / A)$ and per height $(d V / d t / h)$}

In Figure 4 all plots show a decrease in the evaporation rate with time (including the drop not covered by the dome, namely open to free air). The evaporation rate decrease for drops in free air is due to the decrease in the drop's size, while for the drops under the dome the decrease in $d V / d t$ is primarily due to increase in humidity under the dome. To decouple the two phenomena we normalize the evaporation rate by the drop's size (considering height and area as "sizes"). Thus we plot in Figure 5 and 6, curves of $(d V / d t / A)$ and $(d V / d t / h)$ respectively, where $A$ is the area of the drop liquid-vapor interface and $h$ the drop's height. Comparing Figures 5a, 5b, and 5c reveals that central drops surrounded by satellite drops of the same initial total surface area (Figure 5b) 
have the same evaporation flux within the experimental error, as opposed to those surrounded by the same initial total satellite drops volume (Figure 5a) or same initial sum of drop heights (Figure 5c). Observing the drops evaporation at ambient conditions (without dome) we learn from Figures 5 and 6 that the evaporation rate follows equation (3), namely it is proportional to the drop's height. We also see that while the domed drops which are surrounded by satellites reach steady state in about $2-3$ minutes, the domed single drops take longer, and the open to air drops reach steady state instantaneously because they are maintained at the same humidity conditions (Figure 6).

Figures 5 and 6 show that on one hand the drop evaporation rate is proportional to the drop's height (as predicted by Rowan et al.'s equation) and on the other hand the evaporation suppression is proportional to the system's total drops' liquid-vapor area (and not their heights). Note that there is no fundamental difference between the "central" drop and a "satellite" drop they evaporate and suppress each other's evaporation according to the same rules. Thus, our experiments show that although a drop's evaporation rate is proportional to its height, the vapor pressure it produces inside the dome is proportional or closer to proportionality to its total liquidvapor surface area. 

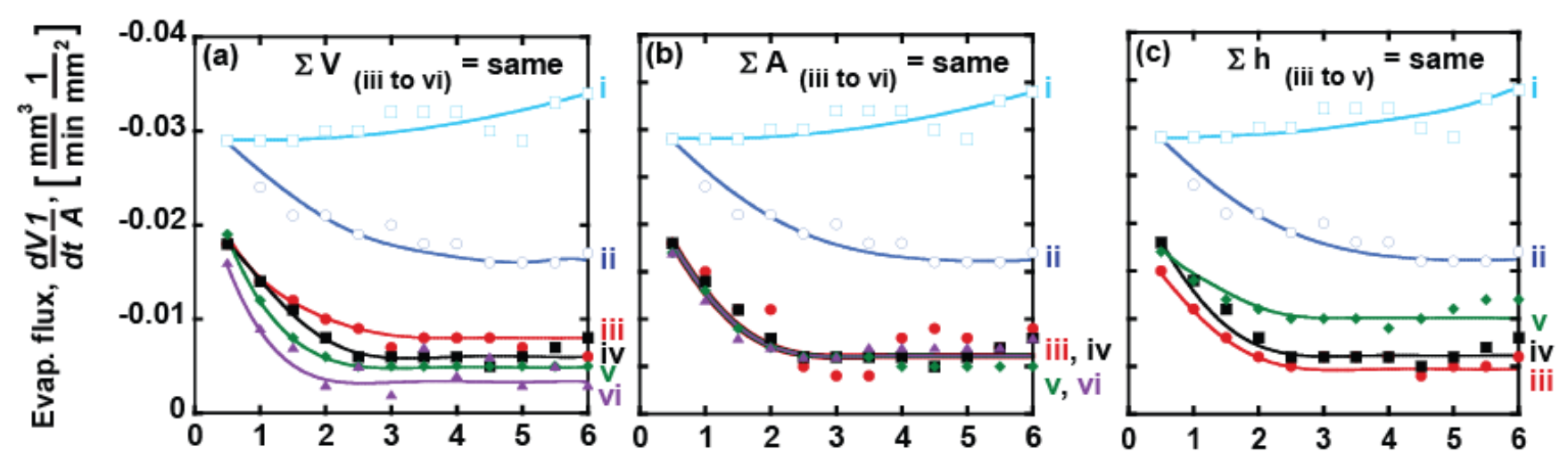

Time from drop placement, $t$, [min]

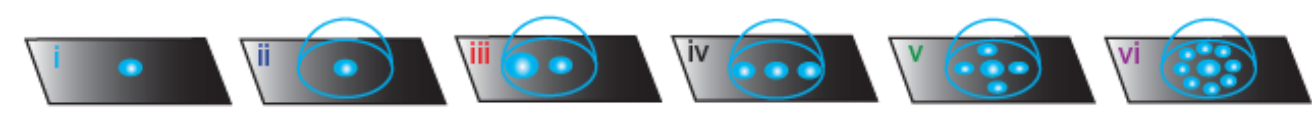

Figure 5. The evaporation flux, $\mathrm{d} V / \mathrm{d} t / A$, of a water drop on Silanized Silicon as a function of time when the drop is in: $(\square)$ Ambient air; (O) under the dome with no satellites; $(\bullet),(\mathbb{\square}),(\diamond)$, (A) under the dome with 1, 2, 4 and 8 satellite drops respectively. The satellite drops have the same initial total sum of (a) Volume of $2 \mu \mathrm{L}$ (e.g. one satellite drop of $2 \mu \mathrm{L}$ or eight satellite drops of $1 / 4 \mu \mathrm{L}$ each). (b) Surface area of $7.7 \mathrm{~mm}^{2}$. (c) Heights of $1.6 \mathrm{~mm}$. The solid lines are guides to eye. The schematic of satellite drops under the dome are just for illustration purposes. In reality, the dome covers the whole surface as shown in Figure 1.

Observing the plateau of the domed and satellited drops in Figures 5a and 5c (and similarly in Figures 6a and 6c), we see that the order of curves iii, iv, v, and vi is opposite in the two figures. The two cases correspond to keeping the same sum of volumes (Figures 5a and 6a) or heights (Figures 5c and 6c). The dependencies of drops size to the power of 1 (height) and 3 (volume) are opposite suggesting that the true parameter to control the evaporation rate is drops size to the power of 2 or close to that power (area as shown in Figures $5 b$ and $6 b$ ). This may seem intuitive, but currently there is no theory to support it, while there are theories to support evaporation rate being proportional to the drop height. 

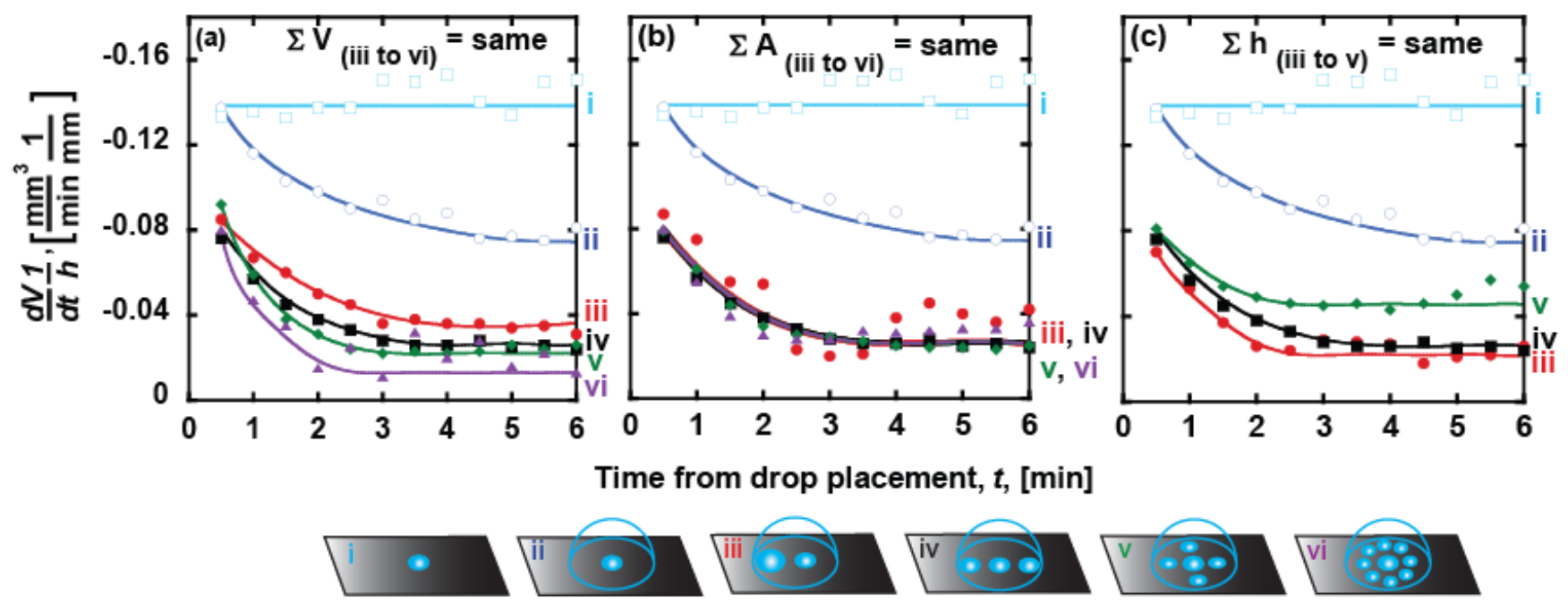

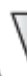
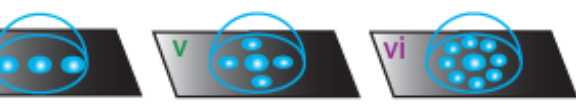

Figure 6. The evaporation rate per height, $\mathrm{d} V / \mathrm{d} t / h$, of a water drop on Silanized Silicon as a function of time when the drop is in: $(\square)$ Ambient air; (O) under the dome with no satellites; ( $\bullet$ ), $(\square),(\diamond),(\boldsymbol{\Delta})$ under the dome with 1, 2, 4 and 8 satellite drops respectively. The satellite drops have the same initial total sum of (a) Volume of $2 \mu \mathrm{L}$ (e.g. one satellite drop of $2 \mu \mathrm{L}$ or eight satellite drops of $1 / 4 \mu \mathrm{L}$ each). (b) Surface area of $7.7 \mathrm{~mm}^{2}$. (c) Heights of $1.6 \mathrm{~mm}$. The solid lines are guides to eye. The schematic of satellite drops under the dome are just for illustration purposes. In reality, the dome covers the whole surface as shown in Figure 1.

\section{Relative Humidity as a function of time}

By fitting equations (2) and (3) to our experimental data we can calculate the relative humidity for a drop in ambient air and drops inside the dome respectively. The results of these calculations at different times are shown in Figure 7. For ambient drops, as expected, we find a constant relative humidity, which agrees well with the humidity shown as light blue straight line at 36\% which represents an independent measurement using a hygrometer. We use this measurement as a confirmation to equations (2) and (3) and use equation (3) to calculate relative humidities for the case where there is no room to place a hygrometer, i.e. inside the dome. For our geometry, the difference in the calculated relative humidity using equation (2) or (3) is negligible. For the 
domed drops, Figure 7 shows that the relative humidity is a function of the drops' total surface area. Again we see that while the evaporation rate is proportional to the drops sum of heights, the relative humidity that they produce is proportional to their total area.

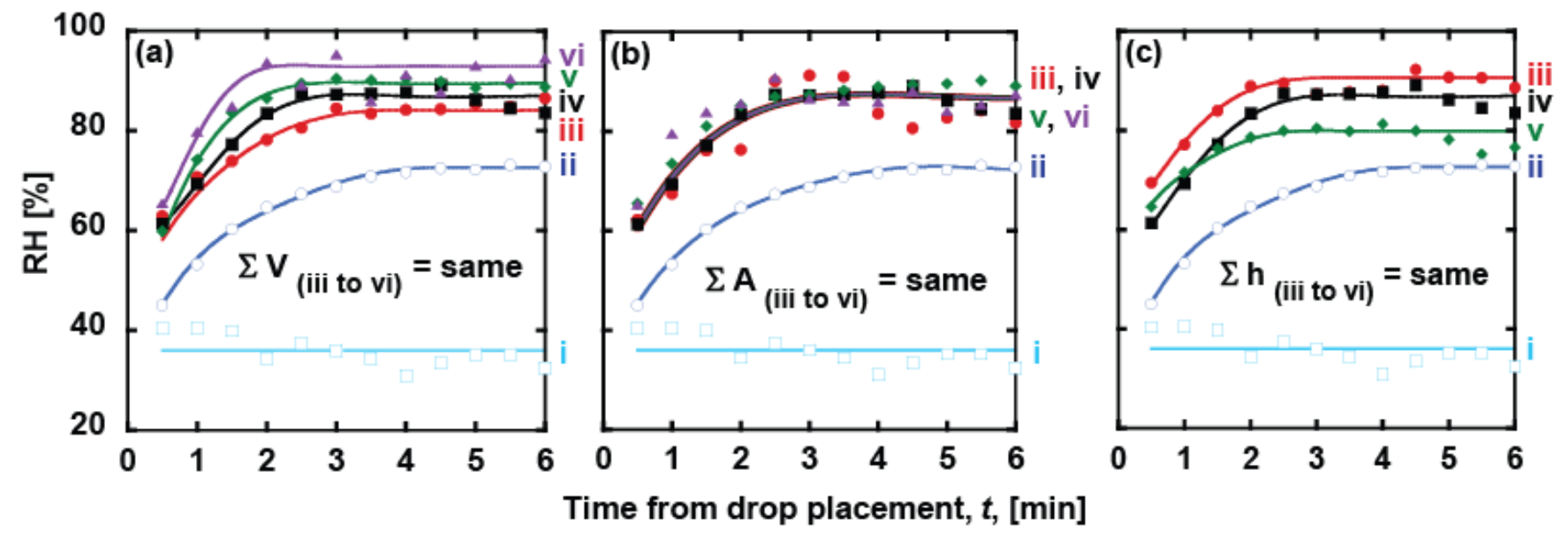

\section{$\underline{\text { Discussion }}$}

Why on one hand the evaporation rate is proportional to the drops heights and yet the resultant humidity is proportional to the liquid-air surface area? To understand this, we remind that the Maxwell equation emanates from the integration of the following diffusion equation.

$$
\frac{d V}{d t}=-\frac{D}{\rho} \frac{d C}{d r}\left(4 \pi r^{2}\right)
$$


The Rowan et al. equation emanates from a similar integration, but instead of integrating over a sphere area $\left(4 \pi r^{2}\right)$ the integration is only over a spherical cap area $\left(2 \pi r^{2}(1-\cos \theta)\right.$ :

$\frac{d V}{d t}=-\frac{D}{\rho} \frac{d C}{d r}\left(2 \pi r^{2}(1-\cos \theta)\right)$

where $C$ is the liquid-vapor concentration for the drop, $r$ is a radial coordinate from the center of the drop (c.f. Figure 8), (and $2 \pi r^{2}(1-\cos \theta)$ is the spherical cap area available for diffusion over the evaporating drop).

Integrating equations (4) or (5) in a three dimensional space, as in a drop surrounded by infinite air (equation (4)), or in a semi-infinite space as in equation (5) results in Maxwell or Rowan et al. equations respectively. In these cases the effect of the drop on the humidity is negligible. However, when evaporation is suppressed by blocking most airways such as by covering the drop with a hemispherical dome, the rate of evaporation is still proportional to the drop height (c.f. equation (3)) but the resultant humidity around the drop is now not a function of $2 \pi r^{2}(1-\cos \theta)$ since it is no longer the area of the envelope available for dissipation of the evaporating water. Now, the dissipation of the vapor is through the area of the airways that is not blocked by the dome. That area is not a function of $r$, and does not have a radial geometry. Hence the resultant humidity is not a function of $h$.

Thus, $\left(\rho \frac{d V}{d t}\right)$ is still a function of $h$, but the humidity in the dome is not. The humidity in the dome is determined by the rate at which two areas exchange vapor: one area is the total area of the drops, the other area is the airways area not blocked by the dome. Therefore, the differences in the plateau values in figure 7 represent different ratios of the total evaporating area to the total area available for leakage. The leakage area is the same for all plots, but the drops evaporating area differs if we use different configurations of the same initial total volume or the same initial 
sum of drop heights. Only when we keep different configurations with the same total initial drop area (figure $7 b$ ) we get a similar evaporation rate for the different experiments.

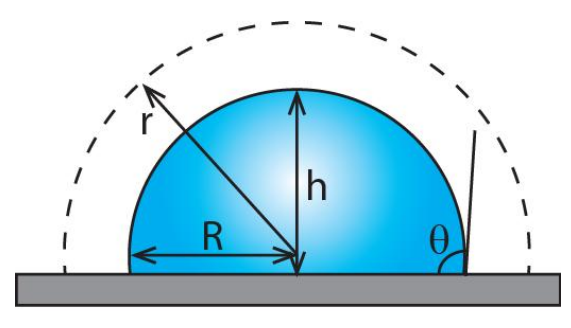

Figure 8. Schematic of evaporating drop used as basis for Maxwell and Rowan et al.'s derivation: the drop height $(h)$, drop's radius of curvature $(R)$, contact angle $(\theta)$ and $(r)$ radial coordinate delineating the envelop boundaries.

\section{SUMMARY}

We show an effective and easy way to induce arbitrary and specific humidity conditions inside a chamber by utilizing either incidental or deliberate small leakages in the system. If the chamber is properly sealed, the humidity approaches $100 \%$ and no variation of the humidity is possible. But, if the chamber is loosely closed, it is easy to create a vapor balance that allows producing specific humidity values. Earlier methods ${ }^{19,20,22,24}$ to control humidity relied on some form of additional salt solution that introduced yet another chemical to the system (the salt) and required an additional setup and constant convection (via a pump). With the method described here, we overcome the need for convection, and allow for the first time to control humidity or suppress evaporation without applying wind.

The incoming vapor is induced by small drops added into the chamber and act as steam suppliers. The outgoing vapor stream is induced by leakages from the chamber to the room which is at a lower relative humidity. The humidity inside the chamber can be accurately determined from the rate of evaporation of the drops, which obeys the Rowan et al. equation ${ }^{42}$, 
namely the evaporation rate is proportional to the drop's height. However, our experiments show that the actual humidity inside the chamber is determined by the sum of surface areas of the drops (and not by the sum of their heights as seemingly suggested by the Rowan et al. equation). This is apparently the result of the balance between the incoming and outgoing vapor. Thus, on one hand the rate of evaporation of a specific drop is proportional to its height, but on the other hand, the actual vapor pressure (or relative humidity) inside the chamber hosting the drop is proportional to the total surface area at the drops' air-liquid interface.

This is particularly useful for systems in which vapor control is needed, but no convection is required, or that convection should be prevented. Future studies that incorporate the proposed tool with such systems will improve the accuracy of experimental findings in these fields. Examples for such fields include processes such as Marangoni flows where controlling the vapors of the volatile component is essential ${ }^{50}$, for system that require careful drying as in the case of phospholipid films ${ }^{51}$, or for systems that consider solid liquid interactions in which the humidity needs to be controlled as in concrete-water interactions ${ }^{52}$ or interactions of water with hydrophobic microstructures ${ }^{53}$, or any other system that consider small volumes of volatile components ${ }^{33,54-60}$.

\section{Acknowledgements}

The paper was supported by the National Science Foundation Grants CMMI-1405109 and CBET-1428398, and by Entergy.

\section{AUTHOR INFORMATION}

\section{Corresponding Author}

Telephone: (409) 880 7791. Fax: (409) 880 2197. Email: rafael.tadmor@lamar.edu 


\section{REFERENCES}

1. Bajgai, T. R.; Raghavan, G. S. V.; Hashinaga, F.; Ngadi, M. O. Electrohydrodynamic Drying a Concise Overview. Drying Technol. 2006, 24, 905-910.

2. Sun, W. Z.; Lin, W. J.; Alai, M. S. Preparation of Microparticles for Acid-Labile Lansoprazole by Solvent Evaporation Method Combined with a Spray Drying Process. J. Food Drug Anal. 2012, 20, 438-445.

3. Costelloe, B.; Finn, D. Experimental Energy Performance of Open Cooling Towers Used under Low and Variable Approach Conditions for Indirect Evaporative Cooling in Buildings. Building Services Eng. Res. and Tech. 2003, 24, 163-177.

4. Glanville, P.; Kozlov, A.; Maisotsenko, V. Dew Point Evaporative Cooling: Technology Review and Fundamentals. Ashrae Transactions 2011, 111-118.

5. Srivastava, H. P.; Arthanareeswaran, G.; Anantharaman, N.; Starov, V. M. Performance and Properties of Modified Poly (Vinylidene Fluoride) Membranes Using General Purpose Polystyrene (Gpps) by Dips Method. Desalination 2011, 283, 169-177.

6. Caricato, A. P.; Luches, A. Applications of the Matrix-Assisted Pulsed Laser Evaporation Method for the Deposition of Organic, Biological and Nanoparticle Thin Films: A Review. Appl. Phys. A: Mater. Sci. Process. 2011, 105, 565-582.

7. Pai, Y.-H.; Tseng, C.-W.; Lin, G.-R. Size-Dependent Surface Properties of Low-Reflectivity Nanoporous Alumina Thin-Film on Glass Substrate. J. Electrochem. Soc. 2012, 159, E99-E102.

8. Shinbo, K.; Uno, A.; Hirakawa, R.; Baba, A.; Ohdaira, Y.; Kato, K.; Kaneko, F. Fabrication of a Quartz-Crystal-Microbalance/Optical-Waveguide Hybrid Sensor and in Situ Evaluation of Vacuum-Evaporated Lead Phthalocyanine Thin Film. Jpn. J. Appl. Phys. 2013, 52, 5.

9. Meroni, D.; Ardizzone, S.; Cappelletti, G.; Ceotto, M.; Ratti, M.; Annunziata, R.; Benaglia, M.; Raimondi, L. Interplay between Chemistry and Texture in Hydrophobic Tio2 Hybrids. $J$. Phys. Chem. C 2011, 115, 18649-18658.

10. Veres, Á.; Ménesi, J.; Juhász, Á.; Berkesi, O.; Ábrahám, N.; Bohus, G.; Oszkó, A.; Pótári, G.; Buzás, N.; Janovák, L.; Dékány, I. Photocatalytic Performance of Silver-Modified Tio2 Embedded in Poly(Ethyl-Acrylate-Co-Methyl Metacrylate) Matrix. Colloid Polym. Sci. 2014, 292, 207-217.

11. Qiao, W.; Zhang, T.; Yen, T.; Ku, T.-H.; Song, J.; Lian, I.; Lo, Y.-H. Oil-Encapsulated Evaporating Droplet Array for Bio-Molecular Detection. Anal. Biomed. Eng. 2014, in press.

12. Mrabet, B.; Mejbri, A.; Mahouche, S.; Gam-Derouich, S.; Turmine, M.; Mechouet, M.; Lang, P.; Bakala, H.; Ladjimi, M.; Bakhrouf, A.; Tougaard, S.; Chehimi, M. M. Controlled Adhesion of Salmonella Typhimurium to Poly(Oligoethylene Glycol Methacrylate) Grafts. Surf. Interface Anal. 2011, 43, 1436-1443.

13. Squires, T. M.; Messinger, R. J.; Manalis, S. R. Making It Stick: Convection, Reaction and Diffusion in Surface-Based Biosensors. Nat. Biotechol. 2008, 26, 417-426.

14. Niziol, J.; Kobierski, J.; Haranczyk, H.; Zaitacz, D.; Hebda, E.; Pieichowski, J. Hydration Properties of Selected DNA-Lipid Complexes. POLIMERY 2015, 60, 18-25.

15. Scholl, S. K.; Schmidt, S. J. Determining the Mechanism and Parameters of Hydrate Formation and Loss in Glucose. Journal of food science 2014, 79, E2232-E2244.

16. Varghese, K. S.; Radhakrishna, K.; Bawa, A. Moisture Sorption Characteristics of Freeze Dried Whey-Grape Beverage Mix. Journal of food science and technology 2014, 51, 2734-2740. 17. Li, Y.; Chen, Y.-F.; Zhou, C.-B. Hydraulic Properties of Partially Saturated Rock Fractures Subjected to Mechanical Loading. Engineering Geology 2014, 179, $24-31$. 
18. Baquerizo, L. G.; Matschei, T.; Scrivener, K. L.; Saeidpour, M.; Thorell, A.; Wadso, L. Methods to Determine Hydration States of Minerals and Cement Hydrates. Cement and Concrete Research 2014, 65, 85-95.

19. Forney, C. F.; Brandl, D. G. Control of Humidity in Small Controlled-Environment Chambers Using Glycerol-Water Solutions. HortTechnology 1992, 2, 52-54.

20. Cao, Y.; Chen, Y.; Mu, T. A New Way to Tune Relative Humidity: By Saturated Ionic Liquid Aqueous Solutions. New Journal of Chemistry 2013, 37, 3890-3898.

21. Carotenuto, A.; Dell'Isola, M. An Experimental Verification of Saturated Salt Solution-Based Humidity Fixed Points. International journal of thermophysics 1996, 17, 1423-1439.

22. Pollio, M.; Kitic, D.; Resnik, S. Research Note: Awvalues of Six Saturated Salt Solutions at $25^{\circ} \mathrm{C}$. Re-Examination for the Purpose of Maintaining a Constant Relative Humidity in Water Sorption Measurements. LWT-Food Science and Technology 1996, 29, 376-378.

23. Charola, A. E. Salts in the Deterioration of Porous Materials: An Overview. Journal of the American institute for conservation 2000, 39, 327-343.

24. Goncalves, T. D.; Abreu, M. M. Testing of Salt-Contaminated Materials: Use of Aqueous Solutions for Control of Relative Humidity. Journal of Testing and Evaluation 2007, 35, 529.

25. Picknett, R. G.; Bexon, R. The Evaporation of Sessile or Pendant Drops in Still Air. J. Colloid Interface Sci. 1977, 61, 336-350.

26. Bormashenko, E.; Musin, A.; Zinigrad, M. Evaporation of Droplets on Strongly and Weakly Pinning Surfaces and Dynamics of the Triple Line. Colloids Surf., A 2011, 385, 235-240.

27. Birdi, K. S.; Vu, D. T.; Winter, A. A Study of the Evaporation Rates of Small Water Drops Placed on a Solid Surface. J. Phys. Chem. 1989, 93, 3702-3703.

28. Nguyen, T. A. H.; Nguyen, A. V.; Hampton, M. A.; Xu, Z. P.; Huang, L. B.; Rudolph, V. Theoretical and Experimental Analysis of Droplet Evaporation on Solid Surfaces. Chem. Eng. Sci. 2011, 69, 522-529.

29. Belman, N.; Jin, K.; Golan, Y.; Israelachvili, J. N.; Pesika, N. S. Origin of the Contact Angle Hysteresis of Water on Chemisorbed and Physisorbed Self-Assembled Monolayers. Langmuir 2013, 28, 14609-14617.

30. Erbil, H. Y. Evaporation of Pure Liquid Sessile and Spherical Suspended Drops: A Review. Adv. Colloid Interface Sci. 2012, 170, 67-86.

31. Yu, Y.-S.; Wang, Z.; Zhao, Y.-P. Experimental and Theoretical Investigations of Evaporation of Sessile Water Droplet on Hydrophobic Surfaces. Journal of Colloid and Interface Science 2012, 365, 254-259.

32. Yu, Y.-S.; Wang, Z.-Q.; Zhao, Y.-P. Experimental Study of Evaporation of Sessile Water Droplet on Pdms Surfaces. Acta Mechanica Sinica 2013, 29, 799-805.

33. Tadmor, R.; Bahadur, P.; Leh, A.; N'guessan, H. E.; Jaini, R.; Dang, L. Measurement of Lateral Adhesion Forces at the Interface between a Liquid Drop and a Substrate. Physical Review Letters 2009, 103, 266101.

34. Tadmor, R. Drops That Pull Themselves Up. Surface Science 2014, 628, 17-20.

35. Xu, W.; Leeladhar, R.; Kang, Y. T.; Choi, C.-H. Evaporation Kinetics of Sessile Water Droplets on Micropillared Superhydrophobic Surfaces. Langmuir 2013, 29, 6032-6041.

36. Barnes, G. T. The Potential for Monolayers to Reduce the Evaporation of Water from Large Water Storages. Agric. Water Management 2008, 95, 339-353.

37. Roberts, W. J. Evaporation Suppression from Water Surfaces. 1957, 38, 740-744.

38. N'Guessan, H. E.; Leh, A.; Cox, P.; Bahadur, P.; Tadmor, R.; Patra, P.; Vajtai, R.; Ajayan, P. M.; Wasnik, P. Water Tribology on Graphene. Nat. Commun. 2012, 3, 1242. 

Retention Force as a Function of Resting Time. Langmuir 2008, 24, 9370-9374.

40. Fuchs, N. A. Evaporation and Droplet Growth in Gaseous Media. Pergamon Press 1959, 1-4. 41. Maxwell, J. C. Collected Scientific Papers. Cambridge Press 1890, 625.

42. Rowan, S. M.; Newton, M. I.; McHale, G. Evaporation of Microdroplets and the Wetting of Solid Surfaces. J. Phys. Chem. 1995, 99, 13268-13271.

43. Fang, X. H.; Li, B. Q.; Petersen, E.; Ji, Y.; Sokolov, J. C.; Rafailovich, M. H. Factors Controlling the Drop Evaporation Constant. J. Phys. Chem. B 2005, 109, 20554-20557.

44. McHale, G.; Rowan, S. M.; Newton, M. I.; Banerjee, M. K. Evaporation and the Wetting of a Low-Energy Solid Surface. J. Phys. Chem. B 1998, 102, 1964-1967.

45. Ninham, B. W.; Nostro, P. L. Molecular Forces and Self Assembly: In Colloid, Nano Sciences and Biology. Cambridge University Press 2010, 13.

46. Kern, W. The Evolution of Silicon Wafer Cleaning Technology. J. Electrochem. Soc. 1990, 1887-1892.

47. Reinhardt, K. A.; Kern, W. Handbook of Silicon Wafer Cleaning Technology (2nd Edition). William Andrew Publishing 2008, 308-316.

48. Einati, H.; Mottel, A.; Inberg, A.; Shacham-Diamand, Y. Electrochemical Studies of SelfAssembled Monolayers Using Impedance Spectroscopy. Electrochim. Acta 2009, 54, 6063-6069. 49. Note that keeping the same total geometric property (volume or surface area or height) of satellite drops means keeping that property the same for all drops.

50. Dai, Q.; Huang, W.; Wang, X. Surface Roughness and Orientation Effects on the ThermoCapillary Migration of a Droplet of Paraffin Oil. Experimental Thermal and Fluid Science 2014, 57, 200-206.

51. Jurak, M. Contact Angle Hysteresis and Phase Separation in Dry Phospholipid Films with Cholesterol Deposited on Mica Surface. Applied Surface Science 2014, 328, 596-605.

52. Ramachandran, R.; Sobolev, K.; Nosonovsky, M. Dynamics of Droplet Impact on Hydrophobic/Icephobic Concrete with Potential for Superhydrophobicity. Langmuir 2015,

53. Lee, J. M.; Lee, S.-h.; Ko, J. S. Dynamic Lateral Adhesion Force of Water Droplets on Microstructured Hydrophobic Surfaces. Sensors and Actuators B: Chemical 2015, 213, 360-367. 54. Bormashenko, E. Y., Wetting of Real Surfaces; Walter de Gruyter, 2013; 19, All.

55. Cho, K.; Wu, A. H.-F.; Liaw, I. I.; Cookson, D.; Lamb, R. N. Wetting Transitions on Hierarchical Surfaces. The Journal of Physical Chemistry C 2015, 116, 26810-26815.

56. Dirany, M.; Dies, L.; Restagno, F.; Leger, L.; Poulard, C.; Miquelard-Garnier, G. Chemical Modification of Pdms Surface without Impacting the Viscoelasticity: Model Systems for a Better Understanding of Elastomer/Elastomer Adhesion and Friction. Colloids and Surfaces A: Physicochemical and Engineering Aspects 2015, 468, 174-183.

57. Hasan, M.; Schroers, J.; Kumar, G. Functionalization of Metallic Glasses through Hierarchical Patterning. Nano Letters 2015,

58. Hemmati, F.; Garmabi, H.; Modarress, H. Effects of Organoclay on the Compatibility and Interfacial Phenomena of Pe/Eva Blends with Ucst Phase Behavior. Polymer Composites 2015, 35, 2329-2342.

59. Israelachvili, J. N., Intermolecular and Surface Forces: Revised Third Edition; Academic press, 2011; All.

60. Tadmor, R. Misconceptions in Wetting Phenomena. Langmuir 2013, 29, 15474-15475. 


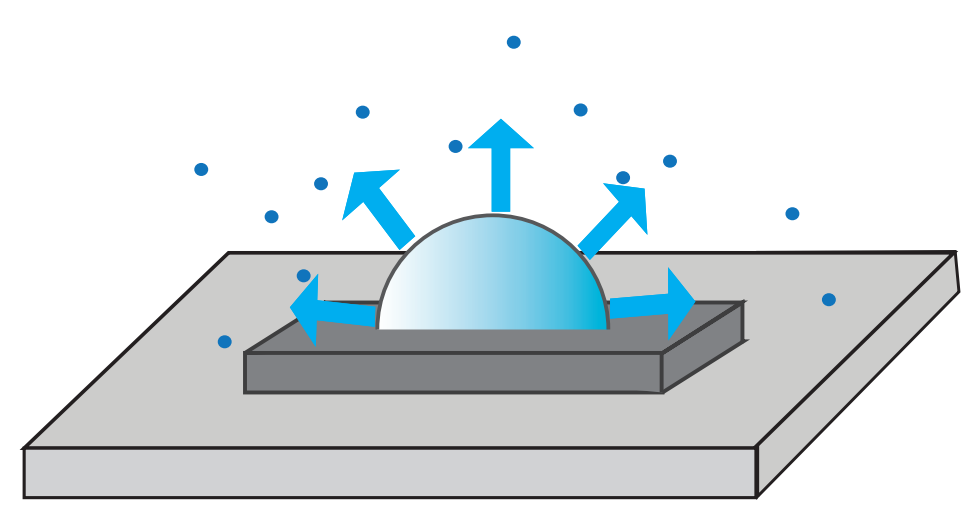

Uncontrolled

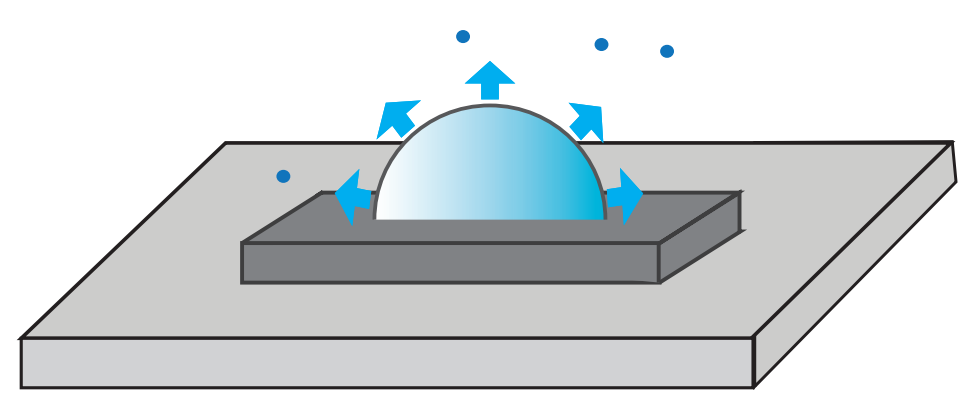

Controlled 